\title{
Immune-related biomarkers in triple-negative breast cancer
}

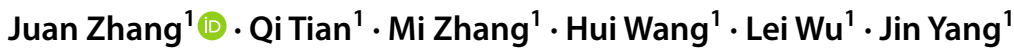 \\ Received: 31 December 2020 / Accepted: 1 April 2021 / Published online: 9 April 2021 \\ (c) The Author(s) 2021
}

\begin{abstract}
Breast cancer is a commonly diagnosed female cancer in the world. Triple-negative breast cancer (TNBC) is the most dangerous and biologically aggressive subtype in breast cancer which has a high mortality, high rates of relapse and poor prognosis, representing approximately $15-20 \%$ of breast cancers. TNBC has unique and special biological molecular characteristics and higher immunogenicity than other breast cancer types. On the basis of molecular features, TNBC is divided into different subtypes and gets various treatments. Especially, immunotherapy becomes a promising and effective treatment to TNBC. However, not all of the TNBC patients are sensitive to immunotherapy, the need of selecting the patients suitable for immunotherapy is imperative. In this review, we discussed recent discoveries about the immune-related factors of TNBC, including tumor-infiltrating lymphocytes (TILs), programmed death-ligand protein-1 (PD-L1), immune gene signatures, some other emerging biomarkers for immunotherapy effectivity and promising biomarkers for immunotherapy resistance. In addition, we summarized the features of these biomarkers contributing to predict the prognosis and effect of immunotherapy. We hope we can provide some helps or evidences to clinical immunotherapy and combined treatment for TNBC patients.
\end{abstract}

Keywords TNBC $\cdot$ Heterogeneity $\cdot$ Immune-related biomarker $\cdot$ Immunotherapy $\cdot$ Prognosis

\section{Introduction}

Breast cancer is known as the most common cancer and becomes one of the main death reasons in female [1]. It is classified into different subtypes according to the expression of molecular features, which includes Luminal A, Luminal B, HER-2 overexpression and triple-negative breast cancer (TNBC). TNBC is the most aggressive cluster of all breast cancers with a rapid progression, high probabilities of early recurrence, and distant metastasis, making up about 15-20\% of breast cancers [2]. The primary and traditional established approaches to treat TNBC patients are surgery, radiation and chemotherapy with serious toxic and side effects. What's more, those patients with a poor response to neoadjuvant treatment and no pathologic complete response (pCR) especially show a poor prognosis and a high risk of distant

Jin Yang

yangjin@mail.xjtu.edu.cn

Juan Zhang

zhj0623@163.com

1 Department of Medical Oncology, The First Affiliated Hospital of Xi' an Jiaotong University, No. 277 West Yanta Road of Xi' an, Xi' an 710061, People's Republic of China relapse, typically within the first $2-3$ years after initial diagnosis. Therefore, the need of novel and better treatment options is dramatically emerging.

Massively parallel sequencing and other 'omics' technologies have revealed unexpected heterogeneity of TNBC [3]. The understanding of the immune profiles with features of TNBC is clearer than before [4]. TNBC has unique biological molecular characteristics and the immunogenicity is higher than other breast cancer subtypes. Immunotherapeutic approaches have shown great and promising developments in recent studies of breast cancer [5], and several clinical trials have shown that immunotherapy could improve clinical outcomes and prognosis of TNBC patients. For example, in Impassion 130 clinical trial, the patients whose tumors have PD-L1 expression level $\geq 1 \%$ with unresectable locally advanced or metastatic TNBC received atezolizumab plus nab-paclitaxel have a prolonged progression-free survival (PFS) and overall survival (OS) [6]. The immunotherapy becomes a new and effective treatment option to TNBC, but not all of the TNBC patients are sensitive to immunotherapy.

To maximize the benefits and minimize the toxicities of cancer immunotherapy, we need to discriminate the patients who will benefit from and get a better response to immunotherapy from others. The immune-related biomarkers 
become necessary [7]. The high expression level of tumorinfiltrating lymphocytes (TILs), programmed death-ligand protein-1 (PD-L1), highly tumor mutational burden (TMB), microsatellite instability (MSI), and mis-match repair deficiency (MMR) are the features of TNBC [8], which may contribute to TNBC patients suitable and sensitive to immunotherapy. These characteristics could be considered as predictors for the efficacy of immunotherapy in breast cancer [9]. From a meta-analysis, PD-L1-positive, first-line therapy, non-liver metastasis, high TILs and CD8 + T-cell infiltrating levels could predict a better response to immune checkpoint inhibitor (ICI) treatment [10]. However, the practical accuracy and usefulness of immune-related biomarkers which could predict promising therapeutic outcomes in TNBC are still controversial. In this review, we summarized the recent progresses and discoveries about the immune-related factors of TNBC. And we analyzed these novel and appropriate immune-related biomarkers which may contribute to predict the response and efficiency of immunotherapy or resistance to immunotherapy.

\section{Existing and under study immune-related biomarkers}

\section{Tumor-infiltering lymphocytes (TILs) in TNBC}

The vast heterogeneity of TNBC mainly originate from the tumor immune microenvironment, which is associated with tumor cell proliferation and aggressive ability, metastasis and drug resistance [11]. TILs comprising different levels of lymphocyte and monocyte infiltration is the main component in tumor immune microenvironment [3], which can be evaluated in H\&E pathologic sections on the basis of established guidelines [12]. Currently, various evidences in scientific interest and clinical setting both have shown that the density, type, and location of TILs in TNBC exhibit different values for assessing disease prognosis and progression.

First of all, in the quantitative level, X. Yu et al. showed that higher value of total tumor-infiltrating lymphocytes (both intraepithelial and stromal) counts associated with better prognosis (pooled HR 0.88, 95\% CI 0.83-0.94) in TNBC than other breast cancer subtypes in a 17 eligible studies including 12,968 candidates. Whether disease-free survival (DFS) or metastasis-free survival, which is statistically significant $(P<0.0001)$. A long overall survival also was indicated by total TILs, but without statistically significant $(P=0.08)$ [13]. Loi et al. demonstrated that high levels of TILs could significantly predict the rates of distant recurrence, and that each $10 \%$ increase of TILs was associated with a $13 \%$ decrease in relative risk of distant recurrence in a study with 134 TNBC patients [14]. TILs also can be viewed as a predictive factor of response to chemotherapy.
For TNBC patients who received neoadjuvant chemotherapy, high expression levels of immune-related markers or immune infiltration were correlated with higher pCR, lower risk of relapses and better outcomes. Denkert, C., et al. indicated that the percentage of intratumoral lymphocytes was a significant independent parameter for $\mathrm{pCR}$ in both cohorts (training cohort: $P=0.012$; validation cohort: $P=0.001$ ). And the pCR rates of $42 \%$ (training cohort) and $40 \%$ (validation cohort) in lymphocyte-predominant breast cancer (LPBC) were higher than those tumors without any infiltrating lymphocytes with pCR rates of $3 \%$ (training cohort) and 7\% (validation cohort) [15]. In two national randomized clinical trials (ECOG 2197 and ECOG 1199) with 481 evaluable TNBC patients had TILs (sTILs, 80\%; iTILs, $15 \%$ ) using contemporary adjuvant chemotherapy, Adams, S., et al. observed that higher sTIL scores were associated with better prognosis. With the increase of sTILs, the risk of recurrence or death reduce. They also confirmed that sTILs should be as a robust prognostic factor in TNBC [16]. Some other clinical trials which could show the correlation between TILs and outcomes in TNBC immunotherapy which is positive (Table1). In KEYNOTE-086 (a phase II trial), among the patients who received pembrolizumab (PD-1 inhibitor) with high stromal TILs levels had an improved ORR in metastatic TNBC. It means that TILs is emerging as a potentially important biomarker to predict the response of immunotherapy in TNBC [17].These findings and trials provide a strong support for the patients with immunotherapy in TNBC [4].

Secondly, certain subgroups of TILs which contain different cellular composition of the immune infiltration represent different immune response and outcomes. We can regard it as qualitative differences. Various lymphocytes components of TILs induce different types of tumor microenvironment. Diverse forms affect the balance of immune response and escape, leading to different prognosis and outcomes in cancers [13]. On one hand, it can induce immunity, killing tumor cells, inhibiting tumor proliferation and progress. The majority of TILs are prominent CD8 + cytotoxic $\mathrm{T}$ lymphocytes (CTLs), which are the major effector cell type in breast cancer and related to a better prognosis [18]. And other immune cells contribute to a protective effect and favorable outcomes, such as CD4 + T helper, natural killer (NK) cells, M1 macrophages, and dendritic cells (DCs) [19]. On the other hand, the immune system can be suppressed by regulatory $\mathrm{T}$ cells, which further form the immune microenvironment to help the survival of tumor cells and promote carcinogenesis. The higher enrichment scores of macrophages M2, immature DCs, and eosinophils are suggested for a worse OS [20]. Foxp $3+\mathrm{T}$ and PD-1 $+\mathrm{T}$ cells infiltration in tumor cells mediate tumor immune escape and might be a worse prognosis predictor in breast cancer [13, 21]. A high ratio of CTLs to FOXP3 + T cells plays a key 


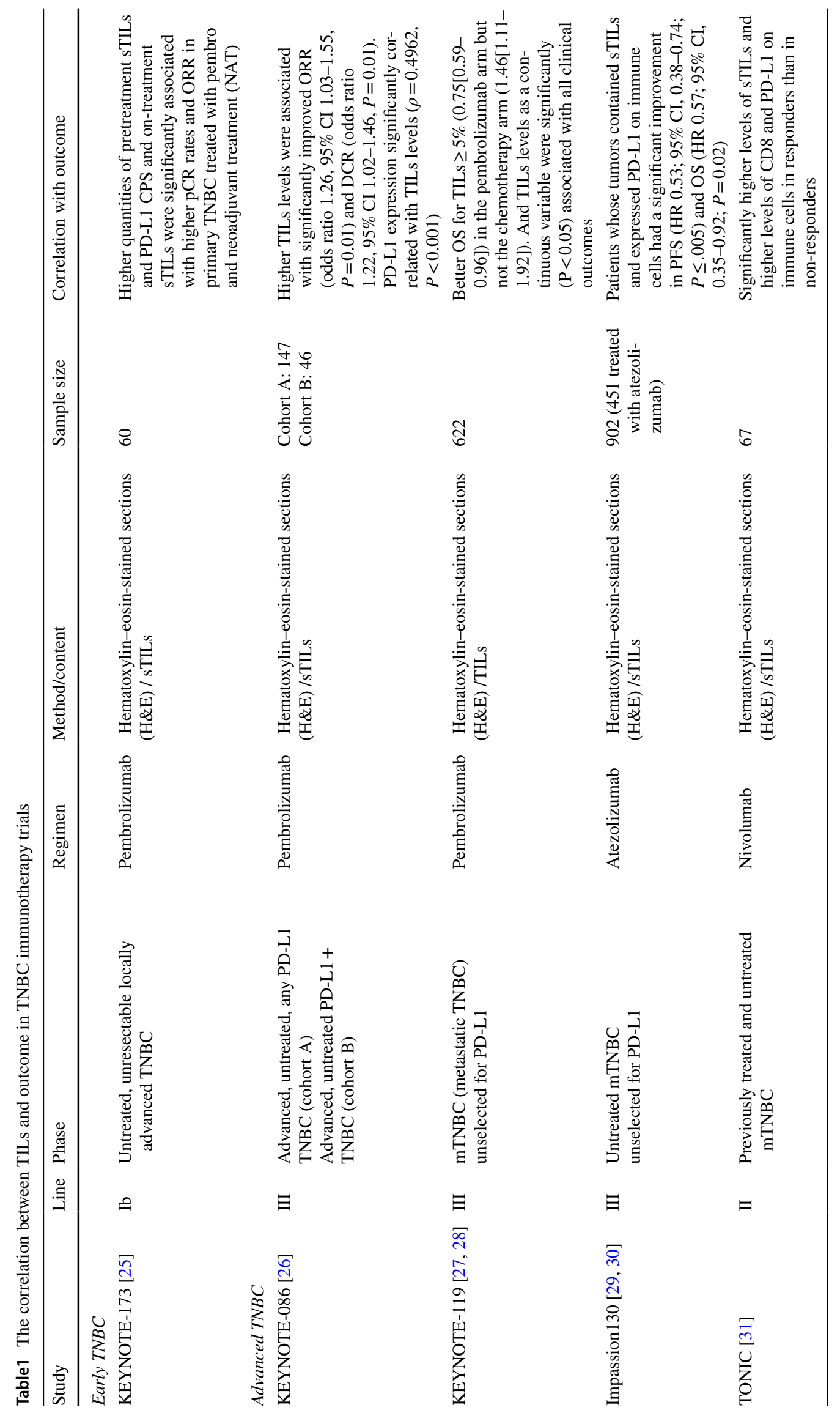


regulatory role in the development and function of $\mathrm{T}$ regulatory cells, which is a strong predictor of $\mathrm{pCR}$ in the neoadjuvant setting [22]. Thirdly, the location of TILs also needs to be considered seriously. Significantly different from other solid tumors, stromal TILs (sTILs) shows a more important and meaningful prognostic value than CTLs (iTILs) in breast cancer [16, 22]. But X. Yu et al. thought that TILs location did not affect the prognosis of breast cancer patients, both iTILs and sTILs contributed to a favorable survival time [13]. In addition, the immunity decreased after tumor metastasis. It means the metastatic lesions had lower TILs and PD-L1 expression levels than primary tumor sites [23]. Perhaps this conclusion could hint at why early immunotherapy is more effective to patients. All in all, TILs have been recognized as the most valuable predictive biomarker, but the accuracy still needs to be confirmed [24]. Whatever, based on previous clinical trials and researches, we can regard it as an important factor related to prognosis.

\section{PD-L1 expression in TNBC}

Programmed cell death receptor ligand 1 gene (PD-L1, also known as CD274 or B7-H1) significantly binds the PD-1 pathway and negatively regulates effector T-cell function [32]. Mittendorf et al. found the expression of PD-L1 is higher in TNBC than other breast cancer subtypes according to The Cancer Genome Atlas (TCGA) RNA sequencing data [33]. A meta-analysis collecting six studies which included 7877 cases has showed that a high expression level of PD-L1 might lead to the mitigation of the host's anti-tumor immune response by activating of the immune checkpoint PD-1/PDL1 pathway, thus resulted in increased tumor aggressiveness [34] including inhibiting T-cell proliferation, promoting immune cell apoptosis, and might help these tumors evade anti-cancer immune responses.

The expression of PD-L1 in different locations induces different outcomes. If PD-L1 is expressed on cancer cells, it means this tumor is more malignant and aggressive, prone to metastasis and have a worse prognosis. A research published in 2014 has shown that high expression of PD-L1 on tumor cells could reflect the immune microenvironment which was associated with an adaptive immune resistance [35]. On the contrary, PD-L1 expressed on TILs showed low-risk clinicopathological parameters and a durable survival time in breast cancer [36]. Recently, Sugie T., et al. reported that PD-L1 expression on tumor cells and on immune cells is significantly correlated with TILs levels and infiltration of CD8 + T cells in TNBC using multiplex fluorescent IHC, which indicated that high level of PD-L1 on IC might reflect T cellinflamed tumors with the amount of TILs present, including the $\mathrm{CD} 8+\mathrm{T}$ cells required for anti-tumor responses [37]. Yuan et al. observed metastatic lymph nodes had higher expression of PD-L1 compared to primary tumor, in a study with 47 paired breast tumor and metastatic axillary lymph node samples [38].

Clinical trials targeting the PD-1/PD-L1 pathway to treat TNBC patients are ongoing, and preliminary results have been promising. The result is a bit different between early TNBC and advanced TNBC with immunotherapy. In early TNBC, patients with PD-L1 positive can get a higher pCR, but no statistically significant difference. With the increase of PD-L1 expression, the $\mathrm{pCR}$ also increases and survival time gets prolonged. Advanced TNBC patients with PD-L1 positive have a better outcome and longer survival time than PD-L1 negative patients. Keynote-522 is a phase 3 trial which added pembrolizumab to neoadjuvant chemotherapy in early TNBC. The conclusion is among all 602 patients, the people who accepted pembrolizumab-chemotherapy obtained a significantly higher percentage of $\mathrm{pCR}$ at the time of definitive surgery than people in the placebo-chemotherapy group. No matter PD-L1 is expressed or not, the combination therapy group always get a longer survival time. But the PD-L1-positive group had a higher pCR [39].

From JAVELIN solid tumor study, a phase 1 study, the metastatic TNBC patients treated with avelumab had higher ORR with a higher expression level of PD-L1 [40]. Keynote-119 showed that the therapeutic effect of pembrolizumab is enhanced with the increase of combined positive score (CPS) [41]. From the Impassion 130 biomarker subgroup analysis, expression of PD-L1 on immune cells was required for response to the combination of atezolizumab plus nab-paclitaxel. In this clinical trial, the patients were evaluated by the expression of PD-L1 on immune cells using SP142 antibody, Ventana. In the ITT analysis, the mPFS was 7.2 months with atezolizumab plus nab-paclitaxel, as compared with 5.5 months with placebo plus nab-paclitaxel. Among patients with PD-L1-positive tumors, the mPFS was 7.5 and 5.0 months, respectively. In the ITT analysis, the median OS was 21.3 months with atezolizumab plus nabpaclitaxel and 17.6 months with placebo plus nab-paclitaxel. Among patients with PD-L1-positive tumors, the median OS was 25.0 and 15.5 months, respectively. It is evident that PFS and OS are longer, as well as the efficiency had been proven in PD-L1 + IC patients (the threshold is PD-L1 > 1\%) [42]. However, the results of the final analysis of IMpassion 130 in ESMO2020 mentioned that while OS differences for atezolizumab + nab-paclitaxel vs placebo + nab-paclitaxel in the IMpassion130 ITT population were not statistically significant, precluding formal testing, clinically meaningful OS benefit was observed in PD-L1 + IC patients (7.5-mo median OS improvement) [43].

Some other trails with immunotherapy also provided evidences about the prognostic and predictive value of PD-L1 expression in breast cancer. We listed them in Table 2. In SABCS2018, a scientifically complex study aimed at determining which patients will have the highest response rates to 


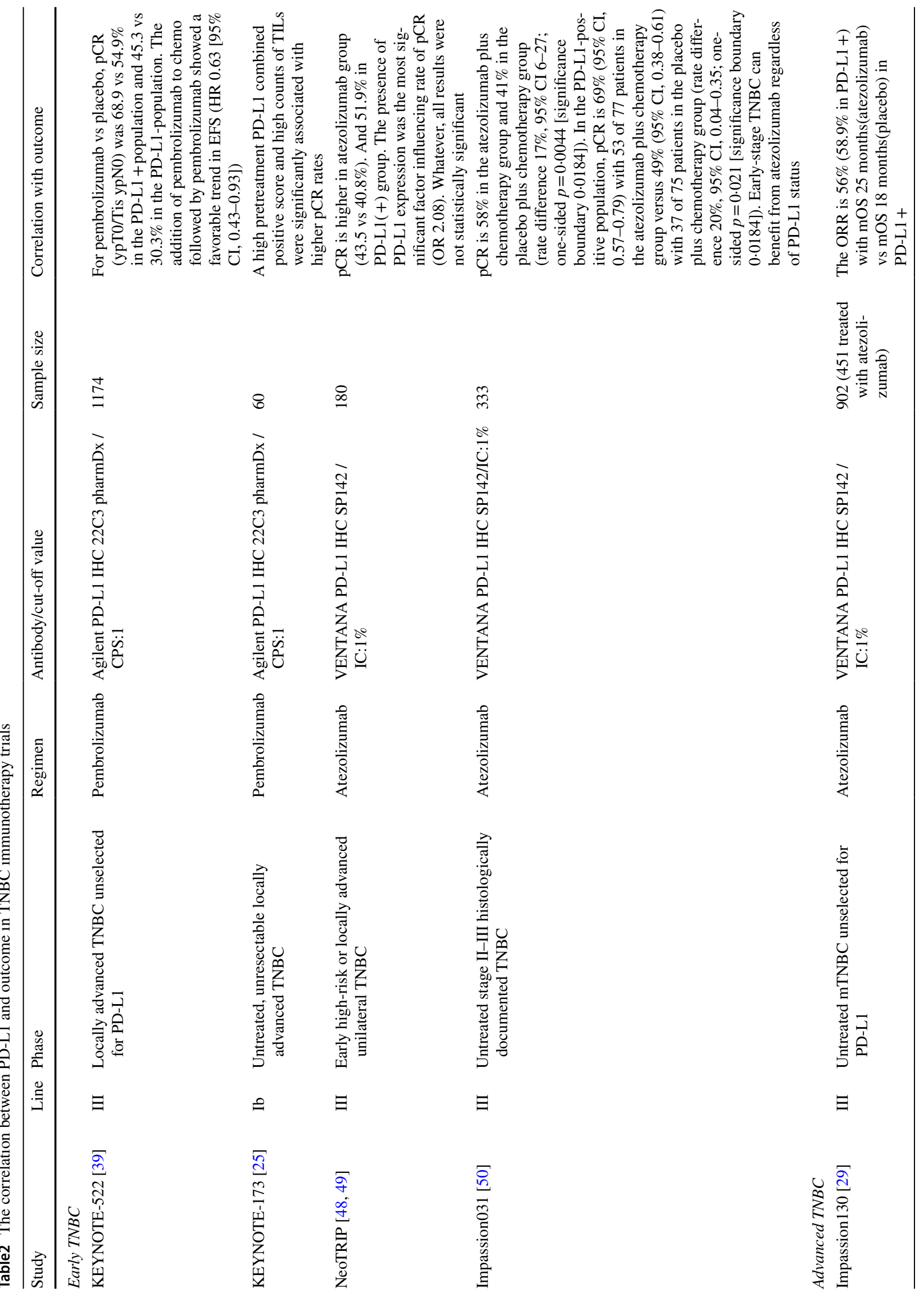




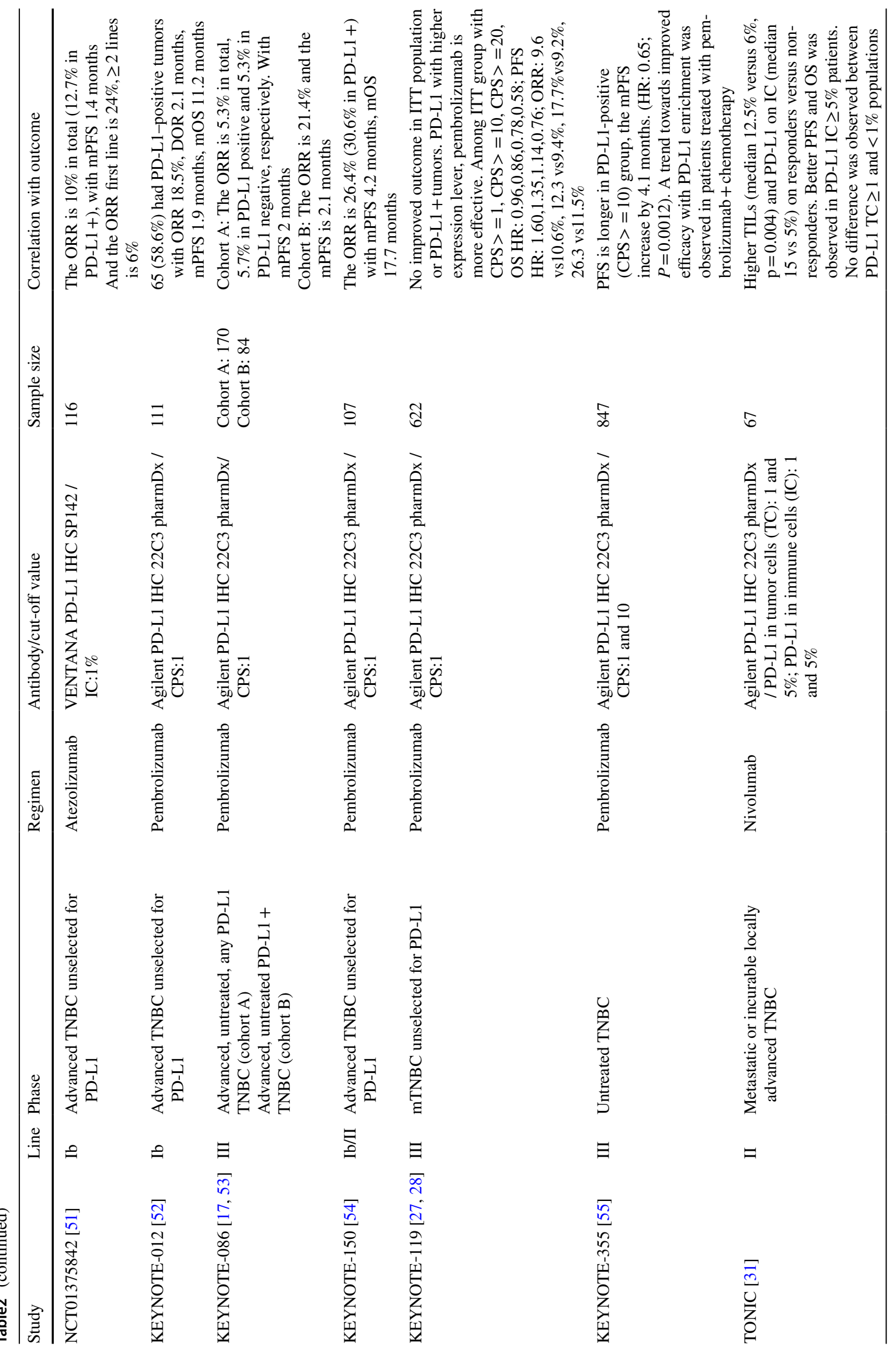




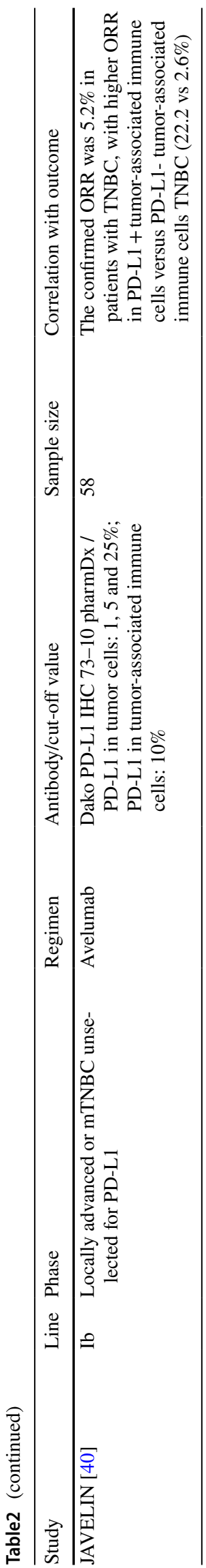

an immunotherapy agent known as atezolizumab, the same agent in the IMPASSION study. That trial showed patients with a marker called PD-L1 benefit from treatment with atezolizumab. In March 2019, atezolizumab plus nab-paclitaxel was approved could be used to treat PD-L1-positive metastatic, locally advanced, or unresectable TNBC by the FDA, based on the data from the Impassion 130 trial. In addition, the Ventana PD-L1 (SP142) assay was also requested as a companion diagnostic device to select TNBC patients for atezolizumab [44].

In short, testing the expression of PD-L1 is of great significance in unresectable locally advanced or metastatic TNBC who might choose immunotherapy. But the criterion of PD-L1 expression level test is still in the dispute. PD-L1 expression is evaluated by using different pathological factors, antibodies, and cut-off points, which may cause various results in different institutions and platforms. The standardized method has not been established and confirmed by a consensus conference in the world. Now in clinical therapy or clinical trials, to test the expression of PD-L1, PD-L1 22C3 (Agilent Technologies Inc., Santa Clara, CA, USA), 28-8 (Agilent Technologies Inc.), SP142 (Roche Tissue Diagnostics, Tucson, AZ, USA), SP263 (Roche Tissue Diagnostics), and 73-10 (Agilent Technologies Inc.) have been taken into consideration [45]. Rugo HS et al. presented a report at ESMO 2019. They tested the PD-L1 status in available samples from IMpassion 130 by using VENTANA SP142 or SP263 IHC assay (IC $\geq 1 \%$, SP142 + or SP263+) or Dako PD-L1 IHC 22C 3 assay (CPS $\geq 1,22 \mathrm{C} 3+$ ). And they found PD-L1 + prevalence was $46 \%$ for SP142+, $81 \%$ for $22 \mathrm{C} 3+$, and $75 \%$ for SP263 + . More patients with PD-L1 + tumors were identified by using $22 \mathrm{C} 3$ and SP263 assays at the evaluated cutoffs. But the patients with PD-L1 + expression tested by SP142 obtained the greatest clinical benefit with atezolizumab plus nab-paclitaxel, which proved that assessment with SP142 helps to extract the most effective population for treatment [46]. Emens LA et al. published a paper to discuss the differences in the evaluation methods of PD-L1 in SP142 staining which assessed PD-L1 IC and TC status. Scoring was based on PDL1-expressing IC as a percentage of tumor area and PD-L1 scoring on TC was based on the percentage of PD-L1-expressing TC. Threshold of both is $1 \%$. They found PD-L1 IC and TC were weakly correlated as continuous variables $(\mathrm{r}=0.26)$. PD-L1 TC + prevalence was low, but most PD-L1 $\mathrm{TC}+$ samples were also PD-L1 IC + . Their analyses on IMpassion 130 patients showed that PD-L1 on TC per se is not associated with the clinical activity of atezolizumab plus nab-paclitaxel. So SP142 assay with testing PD-L1 IC status should be as a clinically validated companion diagnostic for patients with newly diagnosed metastatic TNBC to decide if they could benefit from first-line treatment with atezolizumab plus nab-paclitaxel [30]. In addition, Winer 
EP et al. explained the advantages of CPS which was tested by $22 \mathrm{C} 3$ over other testing methods at SABCS 2020. PD-L1 expression in tumor and immune cells both are important in metastatic TNBC which could be as a predictive biomarker of pembrolizumab efficacy in KEYNOTE-119 cases [47]. So based on the researches published, we prefer the two approaches, SP142 by Ventana and 22C3 by Dako Agilent, which have been approved by FDA [32].TNBC triple-negative breast cancer, PD-L1 programed death-ligand 1, mPFS median progression-free survival, mOS median overall survival, ORR overall response rate, pCR pathological complete response, OR odds ratios, HR hazard ratio.

\section{Immune gene signatures in TNBC}

Different immune cell types express different levels of immune gene signatures with various functions and immune effect mechanisms associated with clinical benefits in TNBC [3].The study collecting 193 TNBC patients have found an inverse association between immune metagene expression and clonal heterogeneity, somatic copy number alteration levels. Lymphocyte-rich TNBCs with better prognosis had significantly lower mutation than lymphocyte-poor TNBCs, which means immune-rich TNBCs were associated with lower clonal heterogeneity. The results were also established in TCGA data set and METABRIC data set [56]. Therefore, it is necessary to develop immune gene signatures into the prognostic and predictive biomarkers in the future treatment for breast cancer [57].

As we know, some factors can be tested from gene levels to evaluate the potential clinical benefits of immunotherapy in cancer. The most multigene assays that have been confirmed were immune checkpoint genes (CTLA-4, IDO1, LAG3, PDCD1, PDL1 and so on) associated to efficiency of ICIs [58]. The expression of PD-L1 is higher in TNBC which contributes to effective responding to immunotherapy as we explained in the last part. Clinically meaningful OS benefit was observed in PD-L1 + patients from the final analysis of IMpassion130 in ESMO2020 [43].

From previous clinical trials and researches, TMB has also been listed to be a predictive biomarker of response to ICIs treatments in different types of cancers [59]. The patients with high TMB were more sensitive and responsive to immunotherapy. But the role of TMB in TNBC remains controversial. Because contrary to other tumors, mutational load is relatively low in breast cancer, suggesting that TMB is unable to contribute to identifying patients benefit from immunotherapy [60]. The present study showed that TMB was not strongly associated with cytolytic activity and different degrees of immune cell infiltration in the immune microenvironment of TNBC which owned the highest expression of immunoregulatory molecules than other kinds of breast cancer [61]. In Impassion130 study, TMB was not regarded as a predictive biomarker of immunotherapy in TNBC. But in KEYNOTE-119 from 2020 ASCO, it was reported that the ORR has been improved in patients with $\mathrm{TMB} \geq 10 \mathrm{mut} /$ $\mathrm{Mb}$, which was $14.3 \%$ in pembrolizumab group vs. $8.3 \%$ in chemotherapy group, compared with $12.7 \%$ in pembrolizumab group vs. $12.8 \%$ in chemotherapy group with $\mathrm{TMB}<10 \mathrm{mut} / \mathrm{Mb}$. The OS also got prolonged in TMB $\geq 10$ mut/Mb population [62].

At the same time, Kraya, A.A., et al. calculated median HRD-total scores which were determined for BRCA1/2, homologous recombination (HR) mutant, and HR wild-type tumors. Then used the scores as the basis for dichotomization for each group of tumors, like homologous recombination repair (HRD)-low group and HRD-high group. They demonstrated that HRD-low TNBC tumors were the most immunogenic subset with high PD1/PD-L1 and TCR signaling, while HRD-high tumors were the least immunogenic in breast cancer [63].

DNA mis-match repair (MMR) system is a type of gene surrogates in tumors. The main reason for tumor is the MMR defect, which leads to the accumulation of genetic sequences of errors, commonly referred to as microsatellites, and presents a microsatellite instability-high (MSI-H) phenotype. MSI-H and mis-match repair deficiency (dMMR) are arguably predictive biomarkers for clinical response to ICIs in solid tumors [64]. Recently, the patients with dMMR or MSI-H across five clinical trials treated with pembrolizumab (KEYNOTE-016, 164, 012, 028, 158) showed a durable response in colorectal, endometrial, biliary, gastric, esophageal, pancreatic and breast cancers [65]. The patients with dMMR could get a longer PFS and OS in colorectal cancer. As well as the responses were durable [66].

Some other new immune gene signatures caught more attentions in recent years. Bernards $\mathrm{R}$, et al. found N-MYC-mediated down-modulation of MHC class I antigen expression [67]. Layer JP, et al. reported a T-cell-poor microenvironment is associated with genomic amplification of the MYCN (N-MYC) proto-oncogene in primary metastatic neuroblastomas [68]. In SABCS2020, Lee JV, et al. reported MYC overexpression is loss of MHC class I in breast cancer, which is related to tumor immune. Tokumaru, Y., et al. established that patients with enrichment of KRAS signaling gene sets were associated with inflammation and favorable tumor immune microenvironment and the patients with KRAS-high owned significantly better DFS and OS than KRAS-low patients in TNBC [69]. Cheng, J.N., et al. demonstrated that TP53 and PIK3CA might be feasible biomarkers to select patients who would benefit most from ICIs by analyzing TCGA database. The patients with the TP53MutPIK3CAWild genotype might improve the response to immunotherapy, which might contribute to precise immunotherapy in TNBC [61]. 


\section{Other biomarkers for breast cancer immunotherapy}

Because tumor tissue biopsy is not easy to get regularly, circulating 'liquid biopsy' biomarkers have been noticed as predictive and prognostic factors which can be non-invasively obtained from patients and trended over time. There are a lot of liquid biomarkers found in predicting the response to immunotherapy. The most biomarkers we know are circulating tumor cells (CTC), cell-free DNA (cfDNA) or circulating tumor DNA (ctDNA) [70], as well as exosomes. Some new blood index are under study, LDH, neutrophil-lymphocyte ratio (NLR), absolute eosinophil count, monocyte count and myeloid-derived suppressor cells (MDSCs), T-cell markers and soluble PD-L1 (sPD-L1), B cell-antibody markers, soluble CD25 (sCD25), blood tumor mutational burden (bTMB) and so on. But all of them were confirmed in nonsmall cell lung carcinoma (NSCLC) or melanoma or oral squamous cell cancer (OSCC) which are still not clear in breast cancer immunotherapy [44]. A relationship between the microbiome and cancer is an ongoing area of research. Gut microbiota is related to the local and systemic innate and adaptive immune responses which may lead to chronic inflammation and cancer. Routy et al. found the outcome of PD-1 blockade could be significantly influenced by gut microbiome in mice and patients. And antibiotic consumption was associated with poor response to immunotherapeutic PD-1 blockade in lung and kidney cancers [71]. In 112 metastatic melanoma patients starting treatment with anti-PD-1 therapy, Gopalakrishnan, V., et al. indicated that the gut microbiome might modulate responses to anti-PD-1 immunotherapy. The patients with a favorable gut microbiome enhanced systemic and anti-tumor immune responses by increasing antigen presentation and improving effector T-cell function in the periphery and the tumor microenvironment [72]. But we do not have an accurate definition and conclusion about the effect of gut microbiome to immunotherapy. Still we should believe that there must be some connections between them.

\section{Biomarkers for immunotherapy resistance}

Not all of the patients respond to immunotherapy. Nonresponders are resistant to immunotherapy when they start to receive treatment called primary resistance. And others generated acquired resistance during therapy or after relapse. Sharma, P., et al. divided resistance into primary and adaptive resistance and explained the mechanisms from tumor cell intrinsic factors and extrinsic factors separately. In the aspect of tumor cell intrinsic factors, the mechanism include absence of antigenic proteins (low mutational burden, lack of viral antigens, lack of cancer-testis antigens, overlapping surface proteins), absence of antigen presentation (deletion in TAP, deletion in B2M, silenced HLA), genetic T-cell exclusion (MAPK oncogenic signaling, stabilized $\beta$-catenin, mesenchymal transcriptome, oncogenic PD-L1 expression) and insensibility to $\mathrm{T}$ cells (mutations in interferon gamma, pathway signaling). To tumor cell extrinsic factors, absence of $\mathrm{T}$ cells (lack of $\mathrm{T}$ cells with tumor, antigen-specific TCRs), inhibitory immune checkpoints (VISTA, LAG-3, TIM-3) and immunosuppressive cells (TAMs, Tregs) were listed [73]. The suppression of anti-tumor immune responses and the progression of cancer were mainly influenced by the increase in the recruitment and activation of immunosuppressive cells, such as Tregs, Bregs, TAMs, and MDSCs within the TME. In addition, the cross-talk between nonimmune cells, for example CAFs and TECs, also contributed to the resistance of immunotherapy [74]. Most of these ultimately are driven by any number of developments: tumor mutations and adaptations, reduced neoantigen generation or expression, disfunction of MHCs, resistance to IFN- $\gamma$ signaling, indoleamine 2,3-dioxygenase (IDO) overexpression, loss of phosphatase and tensin homologue (PTEN) expression, and overexpression of the Wnt $-\beta$-catenin pathway and so on $[75,76]$.

Based on the mechanisms, potential biomarkers are related to immunogenic antigen landscape and preexisting immune context [77]. PD-L1 was regarded as a predictive biomarker for immunotherapy. However, PD-L1-positive patients are not always responsive to immunotherapy and PD-L1-negative people may benefit from it. We cannot just define PD-L1 must be a positive biomarker for immunotherapy. Form another dimension to, it is likely that PD-L1 is in connection with immunotherapy resistance [66]. Because PD-L1 expression can be induced by IFN- $\gamma$, which is related to an active anti-tumor immune response, it was referred to as a mechanism of adaptive immune resistance [73].

Janus kinases (JAK) have been reported related to resistance to immunotherapy. In a mechanistic study, Sucker et al. indicated mutations in JAK 2 could increase IFN- $\gamma$ resistance and reduce subsequent anti-PD-1 therapy failure in melanoma patients [78]. Zaretsky, J.M., et al. analyzed the whole-exome sequencing data based on matched baseline and recurrent biopsy samples from four metastatic melanoma patients who received anti-PD-1 (Pembrolizumab) and experienced initial objective regression of the tumor followed by progression months to years later. They proved immunotherapy resistance-related functional deletion mutation in the gene encoding interferon-receptor-associated JAK1 or JAK2, along with a wild-type allele. Truncation of JAK1 and JAK2 mutations leads to a lack of response to interferon, including insensitivity to its antiproliferative effects on cancer cells [79].

Mutations in beta-2-microglobulin (B2M) disrupt antigen presentation, leading to immune checkpoint blockade therapy resistance [77]. An increase in $\mathrm{B} 2 \mathrm{M}$ mutations was significantly related to an increase in PD-1 + T-cell 
infiltration, indicating that drug resistance caused by B2M mutation is associated with PD-1 + T-cell infiltration [80]. Ishizuka, J.J., et al. showed that loss of function of ADAR1 restores sensitivity to immunotherapy in tumors with a B2M deletion by inactivating antigen presentation by tumor cells [81].

In melanoma, PTEN deletion promotes AKT phosphorylation, thereby promoting PI3K/AKT pathway activation, and ultimately promotes PD-L1 expression, thereby inactivating $\mathrm{T}$ cells. In addition, PTEN inhibits the expression of immunosuppressive factors IL-10, IL-16, and VEGF which may contribute to immunotherapy resistance. The activation of Wnt- $\beta$-catenin signaling pathway was associated with loss of T-cell gene expression characteristics in melanoma. And in colorectal cancer (CRC) tumors can also significantly reduce the infiltration of CD8 + T cells, which might result in resistance to immunotherapy [82]. Additionally, the secretion of inhibitory molecules IDO can have a direct negative effect on T-cell function in the microenvironment [83]. And the combination of IDO inhibitors and immunotherapy has been shown to increase TILs and their functional capacities in the TME.

There are also some other factors which can increase chance for resistance to immune-checkpoint blockers (ICB) immunotherapy, including older age, background infection or chronic disease, smoking, gut microbiota and so on [84]. In conclusion, the mechanisms of resistance to immunotherapy are still under research. But we still can find some way to stimulate immune functions or change the TME to improve the efficiency of immunotherapy, including combined treatment targeting immune and nonimmune targets, chemotherapy, or radiotherapy (Fig. 1).

\section{Results and conclusion}

Immunotherapy is a promising and effective treatment approach to TNBC. Whatever, some patients still cannot respond to immunotherapy. Therefore, it is necessary to identify immune-related biomarkers which can figure out these patients and define risk stratification to achieve accurate treatment. In this review, we listed different immunerelated biomarkers in TNBC, including TILs, PD-L1, immune gene signatures, and other biomarkers, which may be helpful and valuable. Moreover, we explained the promising biomarkers for immunotherapy resistance. As reported, including tumor antigens, TME, signaling pathway, immune molecules and so on also could affect the responses to immunotherapy [45]. TNBC has a high degree of TILs which contribute to a lower risk of disease relapse and a better prognosis than those with a low level of TILs.

At the same time, the treatment of ICIs in metastatic TNBC (mTNBC) with high TILs shows promising results, indicating the potential benefits of immunotherapy for these patients with TNBC [3]. From a research published in 2019, TILs and immune checkpoint molecules were suggested as potential biomarkers to predict the therapeutic efficacy of selected ICIs in TNBC with "immune-inflamed" cluster by Xiao, Y., et al. [85]. The meaning of PD-L1 is still ambiguous because no consistent evaluation standard. Immune gene signatures and other new biomarkers like liquid biopsy and gut microbiome are also still being researched. In addition, the clinical benefits of TNBC patients were improved by the understanding of the impact of different drug components [11]. It is likely that we can use composite biomarkers other than single biomarker to predict clinical outcomes in response of immunotherapy or combined treatment [45]. So
Fig. 1 The potential biomarkers and mechanisms for clinical response or resistance to immunotherapy. (1) PD-L1, programmed cell death receptor ligand $1 ;(2) \mathrm{TME}$, tumor microenvironment; (3) ICs, immune cells, including TAMs (tumor-associated macrophages), Tregs (T regulatory cells), MDSCs (myeloid-derived suppressor cells); non-ICs, nonimmune cells, including CAFs (cancer-associated fibroblasts), TECs (tumor endothelial cells)
Responders

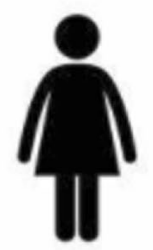

Immunotherapy effectivity
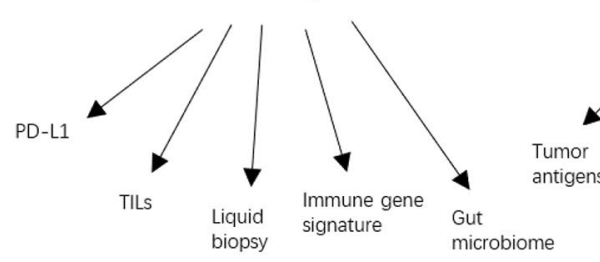

Non-responders
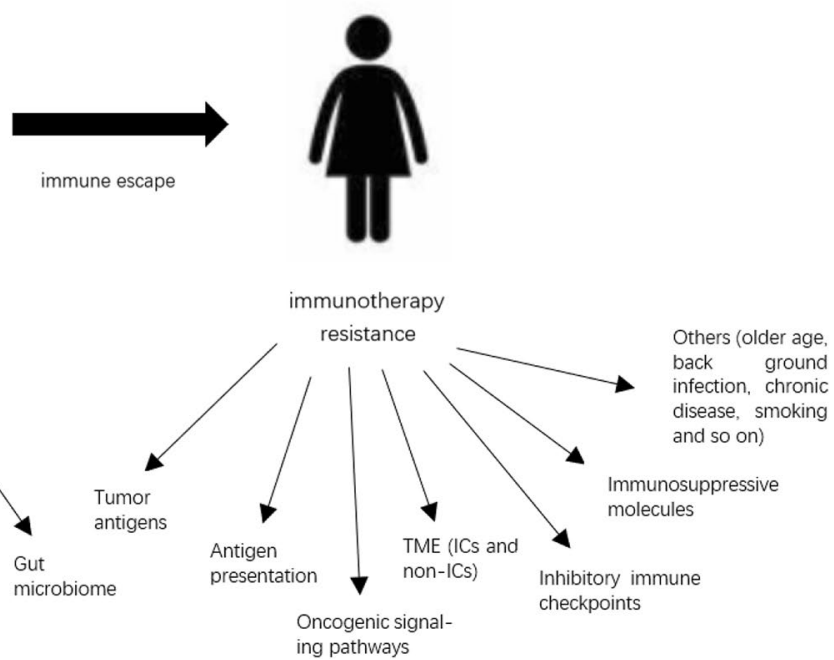
developing advanced methods or using multiple biomarkers to make joint prediction is the focus of future investigation. And in our opinion, we prefer to use PD-L1 and TILs as a more comprehensive composite biomarker. We hope this review can help to recognize the immune-related biomarkers as the important factors to predict response and prognosis for immunotherapy and combined treatment in TNBC.

Funding This study was supported by National Major Scientific and Technological Special Project for "Significant New Drugs Development" (No. 2020ZX09201020) and National Natural Science Foundation of China (No. 82002794).

\section{Declarations}

Conflict of interest The authors declare no conflicts of interest.

Open Access This article is licensed under a Creative Commons Attribution 4.0 International License, which permits use, sharing, adaptation, distribution and reproduction in any medium or format, as long as you give appropriate credit to the original author(s) and the source, provide a link to the Creative Commons licence, and indicate if changes were made. The images or other third party material in this article are included in the article's Creative Commons licence, unless indicated otherwise in a credit line to the material. If material is not included in the article's Creative Commons licence and your intended use is not permitted by statutory regulation or exceeds the permitted use, you will need to obtain permission directly from the copyright holder. To view a copy of this licence, visit http://creativecommons.org/licenses/by/4.0/.

\section{References}

1. Forouzanfar MH, Foreman KJ, Delossantos AM, Lozano R, Lopez AD, Murray CJL, et al. Breast and cervical cancer in 187 countries between 1980 and 2010: a systematic analysis. Lancet. 2011;378(9801):1461-84. https://doi.org/10.1016/S01406736(11)61351-2.

2. Garrido-Castro AC, Lin NU, Polyak K. Insights into molecular classifications of triple-negative breast cancer: improving patient selection for treatment. Cancer Discov. 2019;9(2):176-98. https:// doi.org/10.1158/2159-8290.CD-18-1177.

3. Bianchini G, Balko JM, Mayer IA, Sanders ME, Gianni L. Triplenegative breast cancer: challenges and opportunities of a heterogeneous disease. Nat Rev Clin Oncol. 2016;13(11):674-90. https:// doi.org/10.1038/nrclinonc.2016.66.

4. Jia H, Truica CI, Wang B, Wang Y, Ren X, Harvey HA, et al. Immunotherapy for triple-negative breast cancer: existing challenges and exciting prospects. Drug Resist Updates. 2017;32:115. https://doi.org/10.1016/j.drup.2017.07.002.

5. McArthur HL, Page DB. Immunotherapy for the treatment of breast cancer: checkpoint blockade, cancer vaccines, and future directions in combination immunotherapy. Clin Adv Hematol Oncol. 2016;14(11):922-33.

6. Kang C, Syed YY. Atezolizumab (in combination with nab-paclitaxel): a review in advanced triple-negative breast cancer. Drugs. 2020;80(6):601-7. https://doi.org/10.1007/s40265-020-01295-y.

7. Stovgaard ES, Nielsen D, Hogdall E, Balslev E. Triple negative breast cancer-prognostic role of immune-related factors: a systematic review. Acta Oncol. 2018;57(1):74-82. https://doi.org/ 10.1080/0284186X.2017.1400180.

8. Pusztai L, Karn T, Safonov A, Abu-Khalaf MM, Bianchini G. New strategies in breast cancer: immunotherapy. Clin Cancer Res. 2016;22(9):2105. https://doi.org/10.1158/1078-0432. CCR-15-1315.

9. Marra A, Viale G, Curigliano G. Recent advances in triple negative breast cancer: the immunotherapy era. BMC Med. 2019;17(1):90. https://doi.org/10.1186/s12916-019-1326-5.

10. Zou Y, Zou X, Zheng S, Tang H, Zhang L, Liu P, et al. Efficacy and predictive factors of immune checkpoint inhibitors in metastatic breast cancer: a systematic review and meta-analysis. Ther Adv Med Oncol. 2020;12:1758835920940928. https://doi.org/10. $1177 / 1758835920940928$.

11. Deepak KGK, Vempati R, Nagaraju GP, Dasari VR, Nagini S, Rao $\mathrm{DN}$, et al. Tumor microenvironment: Challenges and opportunities in targeting metastasis of triple negative breast cancer. Pharmacol Res. 2020;153:104683. https://doi.org/10.1016/j.phrs.2020. 104683.

12. Salgado R, Denkert C, Demaria S, Sirtaine N, Klauschen F, Pruneri G, et al. The evaluation of tumor-infiltrating lymphocytes (TILs) in breast cancer: recommendations by an International TILs Working Group 2014. Ann Oncol. 2015;26(2):259-71. https://doi.org/10.1093/annonc/mdu450.

13. Yu X, Zhang Z, Wang Z, Wu P, Qiu F, Huang J. Prognostic and predictive value of tumor-infiltrating lymphocytes in breast cancer: a systematic review and meta-analysis. Clin Transl Oncol. 2016;18(5):497-506. https://doi.org/10.1007/s12094-015-1391-y.

14. Loi S, Michiels S, Salgado R, Sirtaine N, Jose V, Fumagalli D, et al. Tumor infiltrating lymphocytes are prognostic in triple negative breast cancer and predictive for trastuzumab benefit in early breast cancer: results from the FinHER trial. Ann Oncol. 2014;25(8):1544-50. https://doi.org/10.1093/annonc/mdu112.

15. Denkert C, Loibl S, Noske A, Roller M, Muller BM, Komor M, et al. Tumor-associated lymphocytes as an independent predictor of response to neoadjuvant chemotherapy in breast cancer. J Clin Oncol. 2010;28(1):105-13. https://doi.org/10.1200/JCO.2009.23. 7370.

16. Adams S, Gray RJ, Demaria S, Goldstein L, Perez EA, Shulman LN, et al. Prognostic value of tumor-infiltrating lymphocytes in triple-negative breast cancers from two phase iii randomized adjuvant breast cancer trials: ECOG 2197 and ECOG 1199. J Clin Oncol. 2014;32(27):2959-66. https://doi.org/10.1200/jco.2013. 55.0491 .

17. Adams S, Schmid P, Rugo HS, Winer EP, Loirat D, Awada A, et al. Pembrolizumab monotherapy for previously treated metastatic triple-negative breast cancer: cohort A of the phase II KEYNOTE-086 study. Ann Oncol. 2019;30(3):397-404. https://doi. org/10.1093/annonc/mdy517.

18. Seo AN, Lee HJ, Kim EJ, Kim HJ, Jang MH, Lee HE, et al. Tumour-infiltrating CD8+ lymphocytes as an independent predictive factor for pathological complete response to primary systemic therapy in breast cancer. Br J Cancer. 2013;109(10):2705-13. https://doi.org/10.1038/bjc.2013.634.

19. Tower H, Ruppert M, Britt K. The immune microenvironment of breast cancer progression. Cancers. 2019;11(9):1375. https://doi. org/10.3390/cancers11091375.

20. Deng L, Lu D, Bai Y, Wang Y, Bu H, Zheng H. Immune profiles of tumor microenvironment and clinical prognosis among women with triple-negative breast cancer. Cancer Epidemiol Biomark Prev. 2019;28(12):1977. https://doi.org/10.1158/1055-9965. EPI-19-0469.

21. Takenaka M, Seki N, Toh U, Hattori S, Kawahara A, Yamaguchi $\mathrm{T}$, et al. FOXP3 expression in tumor cells and tumor-infiltrating lymphocytes is associated with breast cancer prognosis. Mol Clin Oncol. 2013;1(4):625-32. 
22. Blackley EF, Loi S. Targeting immune pathways in breast cancer: review of the prognostic utility of TILs in early stage triple negative breast cancer (TNBC). Breast. 2019;48:S44-8. https://doi. org/10.1016/s0960-9776(19)31122-1.

23. Esteva FJ, Hubbard-Lucey VM, Tang J, Pusztai L. Immunotherapy and targeted therapy combinations in metastatic breast cancer. Lancet Oncol. 2019;20(3):e175-86. https://doi.org/10.1016/ s1470-2045(19)30026-9.

24. Adams S, Gatti-Mays ME, Kalinsky K, Korde LA, Sharon E, Amiri-Kordestani L, et al. Current landscape of immunotherapy in breast cancer: a review. JAMA Oncol. 2019. https://doi.org/10. 1001/jamaoncol.2018.7147.

25. Schmid P, Salgado R, Park YH, Munoz-Couselo E, Kim SB, Sohn J, et al. Pembrolizumab plus chemotherapy as neoadjuvant treatment of high-risk, early-stage triple-negative breast cancer: results from the phase $1 \mathrm{~b}$ open-label, multicohort KEYNOTE-173 study. Ann Oncol. 2020;31(5):569-81. https://doi. org/10.1016/j.annonc.2020.01.072.

26. Loi S, Adams S, Schmid P, Cortés J, Cescon DW, Winer EP, et al. Relationship between tumor infiltrating lymphocyte (TIL) levels and response to pembrolizumab (pembro) in metastatic triple-negative breast cancer (mTNBC): results from KEYNOTE-086. Ann Oncol. 2017;28:v608. https://doi.org/10.1093/ annonc/mdx440.005.

27. Cortés J, Lipatov O, Im SA, Gonçalves A, Lee KS, Schmid P, et al. KEYNOTE-119: phase III study of pembrolizumab (pembro) versus single-agent chemotherapy (chemo) for metastatic triple negative breast cancer (mTNBC). Ann Oncol. 2019;30:v859-60. https://doi.org/10.1093/annonc/mdz394.010.

28. Loi S, Winer E, Lipatov O, Im S-A, Goncalves A, Cortes J, et al. Abstract PD5-03: Relationship between tumor-infiltrating lymphocytes (TILs) and outcomes in the KEYNOTE-119 study of pembrolizumab vs chemotherapy for previously treated metastatic triple-negative breast cancer (mTNBC). Cancer Res. 2020;80(Suppl 4):PD5-03. https://doi.org/10.1158/1538-7445. SABCS19-PD5-03.

29. Schmid P, Rugo HS, Adams S, Schneeweiss A, Barrios CH, Iwata $\mathrm{H}$, et al. Atezolizumab plus nab-paclitaxel as first-line treatment for unresectable, locally advanced or metastatic triplenegative breast cancer (IMpassion130): updated efficacy results from a randomised, double-blind, placebo-controlled, phase 3 trial. Lancet Oncol. 2020;21(1):44-59. https://doi.org/10.1016/ S1470-2045(19)30689-8.

30. Emens LA, Molinero L, Loi S, Rugo HS, Schneeweiss A, Diéras $\mathrm{V}$, et al. Atezolizumab and nab-paclitaxel in advanced triplenegative breast cancer: biomarker evaluation of the IMpassion130 study. J Natl Cancer Inst. 2021. https://doi.org/10.1093/ jnci/djab004.

31. Voorwerk L, Slagter M, Horlings HM, Sikorska K, van de Vijver $\mathrm{KK}$, de Maaker M, et al. Immune induction strategies in metastatic triple-negative breast cancer to enhance the sensitivity to PD-1 blockade: the TONIC trial. Nat Med. 2019;25(6):920-8. https://doi.org/10.1038/s41591-019-0432-4.

32. Vranic S, Cyprian FS, Gatalica Z, Palazzo J. PD-L1 status in breast cancer: current view and perspectives. Semin Cancer Biol. 2019. https://doi.org/10.1016/j.semcancer.2019.12.003.

33. Mittendorf EA, Philips AV, Meric-Bernstam F, Qiao N, Wu Y, Harrington S, et al. PD-L1 expression in triple-negative breast cancer. Cancer Immunol Res. 2014;2(4):361-70. https://doi.org/ 10.1158/2326-6066.CIR-13-0127.

34. Kim HM, Lee J, Koo JS. Clinicopathological and prognostic significance of programmed death ligand-1 expression in breast cancer: a meta-analysis. BMC Cancer. 2017;17(1):690. https:// doi.org/10.1186/s12885-017-3670-1.

35. Taube JM, Klein A, Brahmer JR, Xu H, Pan X, Kim JH, et al. Association of PD-1, PD-1 ligands, and other features of the tumor immune microenvironment with response to anti-PD-1 therapy. Clin Cancer Res. 2014;20(19):5064-74. https://doi.org/ 10.1158/1078-0432.CCR-13-3271.

36. Huang W, Ran R, Shao B, Li H. Prognostic and clinicopathological value of PD-L1 expression in primary breast cancer: a meta-analysis. Breast Cancer Res Treat. 2019;178(1):17-33. https://doi.org/10.1007/s10549-019-05371-0.

37. Sugie T, Sato E, Miyashita M, Yamaguchi R, Sakatani T, Kozuka Y, et al. Multispectral quantitative immunohistochemical analysis of tumor-infiltrating lymphocytes in relation to programmed death-ligand 1 expression in triple-negative breast cancer. Breast Cancer. 2020;27(4):519-26. https://doi.org/10. 1007/s12282-020-01110-2.

38. Yuan C, Liu Z, Yu Q, Wang X, Bian M, Yu Z, et al. Expression of PD-1/PD-L1 in primary breast tumours and metastatic axillary lymph nodes and its correlation with clinicopathological parameters. Sci Rep. 2019;9(1):14356. https://doi.org/10.1038/ s41598-019-50898-3.

39. Schmid P, Cortes J, Pusztai L, McArthur H, Kümmel S, Bergh $\mathrm{J}$, et al. Pembrolizumab for early triple-negative breast cancer. $\mathrm{N}$ Engl J Med. 2020;382(9):810-21. https://doi.org/10.1056/NEJMo a1910549.

40. Dirix LY, Takacs I, Jerusalem G, Nikolinakos P, Arkenau HT, Forero-Torres A, et al. Avelumab, an anti-PD-L1 antibody, in patients with locally advanced or metastatic breast cancer: a phase 1b JAVELIN Solid Tumor study. Breast Cancer Res Treat. 2018;167(3):671-86. https://doi.org/10.1007/s10549-017-4537-5.

41. Cortés J, Lipatov O, Im SA, Gonçalves A, Lee KS, Schmid P, et al. LBA21-KEYNOTE-119: phase III study of pembrolizumab (pembro) versus single-agent chemotherapy (chemo) for metastatic triple negative breast cancer (mTNBC). Ann Oncol. 2019;30:v859-60. https://doi.org/10.1093/annonc/mdz394.010.

42. Schmid P, Adams S, Rugo HS, Schneeweiss A, Barrios CH, Iwata $\mathrm{H}$, et al. Atezolizumab and nab-paclitaxel in advanced triple-negative breast cancer. N Engl J Med. 2018;379(22):2108-21. https:// doi.org/10.1056/NEJMoa1809615.

43. Emens LA, Adams S, Barrios $\mathrm{CH}$, Dieras VC, Iwata $\mathrm{H}$, Loi $\mathrm{S}$, Rugo HS, Schneeweiss A, Winer EP, Patel S, Henschel V, Swat A, Kaul M, Molinero L, Chui SY, Schmid P. LBA16 - IMpassion130: final OS analysis from the pivotal phase III study of atezolizumab + nab-paclitaxel vs placebo + nab-paclitaxel in previously untreated locally advanced or metastatic triple-negative breast cancer. Ann Oncol. 2020;31(suppl 4):S1142-215.

44. Arora S, Velichinskii R, Lesh RW, Ali U, Kubiak M, Bansal P, et al. Existing and emerging biomarkers for immune checkpoint immunotherapy in solid tumors. Adv Ther. 2019;36(10):2638-78. https://doi.org/10.1007/s12325-019-01051-z.

45. Gonzalez-Ericsson PI, Stovgaard ES, Sua LF, Reisenbichler E, Kos Z, Carter JM, et al. The path to a better biomarker: application of a risk management framework for the implementation of PD-L1 and TILs as immuno-oncology biomarkers in breast cancer clinical trials and daily practice. J Pathol. 2020. https://doi.org/10. 1002/path.5406.

46. Rugo HS, Loi S, Adams S, Schmid P, Schneeweiss A, Barrios $\mathrm{CH}$, et al. LBA20 - performance of PD-L1 immunohistochemistry (IHC) assays in unresectable locally advanced or metastatic triplenegative breast cancer (mTNBC): post-hoc analysis of IMpassion130. Ann Oncol. 2019;30:v858-9. https://doi.org/10.1093/ annonc/mdz394.009.

47. Winer EP, Lipatov O, Im S-A, Goncalves A, Muñoz-Couselo E, Lee KS, et al. Abstract PD14-04: contribution of tumor and immune cells to PD-L1 as a predictive biomarker in triple-negative breast cancer (TNBC): analysis from KEYNOTE-119. Cancer Res. 2021;81(Suppl 4):PD14-04.

48. Gianni L, Huang C-S, Egle D, Bermejo B, Zamagni C, Thill M, et al. Abstract GS3-04: Pathologic complete response (pCR) to 
neoadjuvant treatment with or without atezolizumab in triple negative, early high-risk and locally advanced breast cancer. NeoTRIPaPDL1 Michelangelo randomized study. Cancer Res. 2020;80(Supplement 4):GS3-04.

49. Bianchini G (2020) Tumour infiltrating lymphocytes (TILs), PD-L1 expression and their dynamics in the NeoTRIPaPDL1 trial. Presented at 2020 ESMO Virtual Congress. Sep 19-21, 2020, LBA13

50. Mittendorf EA, Zhang H, Barrios CH, Saji S, Jung KH, Hegg R, et al. Neoadjuvant atezolizumab in combination with sequential nab-paclitaxel and anthracycline-based chemotherapy versus placebo and chemotherapy in patients with early-stage triple-negative breast cancer (IMpassion031): a randomised, double-blind, phase 3 trial. Lancet. 2020;396(10257):1090-100. https://doi.org/10. 1016/s0140-6736(20)31953-x.

51. Emens LA, Cruz C, Eder JP, Braiteh F, Chung C, Tolaney SM, et al. Long-term clinical outcomes and biomarker analyses of atezolizumab therapy for patients with metastatic triple-negative breast cancer: a phase 1 study. JAMA Oncol. 2019;5(1):74-82. https://doi.org/10.1001/jamaoncol.2018.4224.

52. Nanda R, Chow LQ, Dees EC, Berger R, Gupta S, Geva R, et al. Pembrolizumab in patients with advanced triple-negative breast cancer: phase Ib KEYNOTE-012 study. J Clin Oncol. 2016;34(21):2460-7. https://doi.org/10.1200/JCO.2015.64.8931.

53. Adams S, Loi S, Toppmeyer D, Cescon DW, De Laurentiis M, Nanda R, et al. Pembrolizumab monotherapy for previously untreated, PD-L1-positive, metastatic triple-negative breast cancer: cohort B of the phase II KEYNOTE-086 study. Ann Oncol. 2019;30(3):405-11. https://doi.org/10.1093/annonc/mdy518.

54. Tolaney S, Kalinsky K, Kaklamani V, Savulsky C, Olivo M, Aktan G, et al. Abstract PD6-13: phase 1b/2 study to evaluate eribulin mesylate in combination with pembrolizumab in patients with metastatic triple-negative breast cancer. Cancer Res. 2018;78:PD6-13.

55. Cortes J, Cescon DW, Rugo HS, Nowecki Z, Im S-A, Yusof MM, et al. KEYNOTE-355: Randomized, double-blind, phase III study of pembrolizumab + chemotherapy versus placebo + chemotherapy for previously untreated locally recurrent inoperable or metastatic triple-negative breast cancer. J Clin Oncol. 2020;38(suppl 15): 1000

56. Karn T, Jiang T, Hatzis C, Sänger N, El-Balat A, Rody A, et al. Association between genomic metrics and immune infiltration in triple-negative breast cancer. JAMA Oncol. 2017;3(12):1707-11. https://doi.org/10.1001/jamaoncol.2017.2140.

57. Kwon MJ. Emerging immune gene signatures as prognostic or predictive biomarkers in breast cancer. Arch Pharmacal Res. 2019;42(11):947-61. https://doi.org/10.1007/ s12272-019-01189-y.

58. Carbognin L, Pilotto S, Milella M, Vaccaro V, Brunelli M, Caliò A, et al. Differential activity of nivolumab, pembrolizumab and MPDL3280A according to the tumor expression of programmed death-ligand-1 (PD-L1): sensitivity analysis of trials in melanoma, lung and genitourinary cancers. PLoS ONE. 2015;10(6):e0130142. https://doi.org/10.1371/journal.pone.01301 42.

59. Samstein RM, Lee C-H, Shoushtari AN, Hellmann MD, Shen $\mathrm{R}$, Janjigian $\mathrm{YY}$, et al. Tumor mutational load predicts survival after immunotherapy across multiple cancer types. Nat Genet. 2019;51(2):202-6. https://doi.org/10.1038/s41588-018-0312-8.

60. Rizvi NA, Hellmann MD, Snyder A, Kvistborg P, Makarov V, Havel JJ, et al. Cancer immunology. Mutational landscape determines sensitivity to PD-1 blockade in non-small cell lung cancer. Science. 2015;348(6230):124-8.

61. Cheng JN, Ding X, Xu S, Zhu B, Jia Q. Gene expression profiling identified TP53(Mut)PIK3CA(Wild) as a potential biomarker for patients with triple-negative breast cancer treated with immune checkpoint inhibitors. Oncol Lett. 2020;19(4):2817-24. https:// doi.org/10.3892/ol.2020.11381.

62. Winer EP, Lipatov O, Im S-A, Goncalves A, Muñoz-Couselo E, Lee KS, et al. Association of tumor mutational burden (TMB) and clinical outcomes with pembrolizumab (pembro) versus chemotherapy (chemo) in patients with metastatic triple-negative breast cancer (mTNBC) from KEYNOTE-119. J Clin Oncol. 2020;38(suppl 15):1013.

63. Kraya AA, Maxwell KN, Wubbenhorst B, Wenz BM, Pluta $\mathrm{J}$, Rech AJ, et al. Genomic signatures predict the immunogenicity of BRCA-deficient breast cancer. Clin Cancer Res. 2019;25(14):4363. https://doi.org/10.1158/1078-0432. CCR-18-0468.

64. Shen H, Yang ES-H, Conry M, Fiveash J, Contreras C, Bonner JA, et al. Predictive biomarkers for immune checkpoint blockade and opportunities for combination therapies. Genes Dis. 2019;6(3):232-46.

65. Nakamura Y. Biomarkers for immune checkpoint inhibitor-mediated tumor response and adverse events. Front Med (Lausanne). 2019;6:119. https://doi.org/10.3389/fmed.2019.00119.

66. Gibney GT, Weiner LM, Atkins MB. Predictive biomarkers for checkpoint inhibitor-based immunotherapy. Lancet Oncol. 2016;17(12):e542-51. https://doi.org/10.1016/S1470-2045(16) 30406-5.

67. Bernards R, Dessain SK, Weinberg RA. N-myc amplification causes down-modulation of MHC class I antigen expression in neuroblastoma. Cell. 1986;47(5):667-74. https://doi.org/10.1016/ 0092-8674(86)90509-X.

68. Layer JP, Kronmüller MT, Quast T, van den Boorn-Konijnenberg D, Effern M, Hinze D, et al. Amplification of N-Myc is associated with a T-cell-poor microenvironment in metastatic neuroblastoma restraining interferon pathway activity and chemokine expression. Oncoimmunology. 2017;6(6):e1320626. https://doi.org/10.1080/ 2162402x.2017.1320626.

69. Tokumaru Y, Oshi M, Katsuta E, Yan L, Satyananda V, Matsuhashi N, et al. KRAS signaling enriched triple negative breast cancer is associated with favorable tumor immune microenvironment and better survival. Am J Cancer Res. 2020;10(3):897-907.

70. Lee EY, Kulkarni RP. Circulating biomarkers predictive of tumor response to cancer immunotherapy. Expert Rev Mol Diagn. 2019;19(10):895-904. https://doi.org/10.1080/14737159.2019. 1659728

71. Routy B, Le Chatelier E, Derosa L, Duong CPM, Alou MT, Daillère R, et al. Gut microbiome influences efficacy of PD-1based immunotherapy against epithelial tumors. Science. 2018;359(6371):91. https://doi.org/10.1126/science.aan3706.

72. Gopalakrishnan V, Spencer CN, Nezi L, Reuben A, Andrews MC, Karpinets TV, et al. Gut microbiome modulates response to anti-PD-1 immunotherapy in melanoma patients. Science. 2018;359(6371):97. https://doi.org/10.1126/science.aan4236.

73. Sharma P, Hu-Lieskovan S, Wargo JA, Ribas A. Primary, adaptive, and acquired resistance to cancer immunotherapy. Cell. 2017;168(4):707-23. https://doi.org/10.1016/j.cell.2017.01.017.

74. Saleh R, Elkord E. Acquired resistance to cancer immunotherapy: role of tumor-mediated immunosuppression. Semin Cancer Biol. 2020;65:13-27. https://doi.org/10.1016/j.semcancer.2019.07.017.

75. Rieth J, Subramanian S. Mechanisms of intrinsic tumor resistance to immunotherapy. Int J Mol Sci. 2018;19(5):1340. https://doi.org/ 10.3390/ijms 19051340.

76. Lei Q, Wang D, Sun K, Wang L, Zhang Y. Resistance mechanisms of Anti-PD1/PDL1 therapy in solid tumors. Front Cell Dev Biol. 2020;8:672. https://doi.org/10.3389/fcell.2020.00672.

77. Ren D, Hua Y, Yu B, Ye X, He Z, Li C, et al. Predictive biomarkers and mechanisms underlying resistance to PD1/PD-L1 blockade cancer immunotherapy. Mol Cancer. 2020;19(1):19. https:// doi.org/10.1186/s12943-020-1144-6. 
78. Sucker A, Zhao F, Pieper N, Heeke C, Maltaner R, Stadtler N, et al. Acquired IFN $\gamma$ resistance impairs anti-tumor immunity and gives rise to T-cell-resistant melanoma lesions. Nat Commun. 2017;8:15440. https://doi.org/10.1038/ncomms15440.

79. Zaretsky JM, Garcia-Diaz A, Shin DS, Escuin-Ordinas H, Hugo W, Hu-Lieskovan S, et al. Mutations associated with acquired resistance to PD-1 blockade in melanoma. N Engl J Med. 2016;375(9):819-29. https://doi.org/10.1056/NEJMoa1604958.

80. Janikovits J, Müller M, Krzykalla J, Körner S, Echterdiek F, Lahrmann B, et al. High numbers of PDCD1 (PD-1)-positive T cells and mutations in microsatellite-unstable colorectal cancer. Oncoimmunology. 2018;7(2):e1390640. https://doi.org/10.1080/21624 02X.2017.1390640.

81. Ishizuka JJ, Manguso RT, Cheruiyot CK, Bi K, Panda A, IrachetaVellve A, et al. Loss of ADAR1 in tumours overcomes resistance to immune checkpoint blockade. Nature. 2019;565(7737):43-8. https://doi.org/10.1038/s41586-018-0768-9.

82. Wang Z, Wu X. Study and analysis of antitumor resistance mechanism of PD1/PD-L1 immune checkpoint blocker. Cancer Med. 2020. https://doi.org/10.1002/cam4.3410.
83. Kelderman S, Schumacher TNM, Haanen JBAG. Acquired and intrinsic resistance in cancer immunotherapy. Mol Oncol. 2014;8(6):1132-9. https://doi.org/10.1016/j.molonc.2014.07.011.

84. Pitt Jonathan M, Vétizou M, Daillère R, Roberti María P, Yamazaki T, Routy B, et al. Resistance mechanisms to immunecheckpoint blockade in cancer: tumor-intrinsic and -extrinsic factors. Immunity. 2016;44(6):1255-69. https://doi.org/10.1016/j. immuni.2016.06.001.

85. Xiao Y, Ma D, Zhao S, Suo C, Shi J, Xue M-Z, et al. Multi-omics profiling reveals distinct microenvironment characterization and suggests immune escape mechanisms of triple-negative breast cancer. Clin Cancer Res. 2019;25(16):5002. https://doi.org/10. 1158/1078-0432.CCR-18-3524.

Publisher's Note Springer Nature remains neutral with regard to jurisdictional claims in published maps and institutional affiliations. 